\title{
Rheuma und Lifestyle
}

Eine persistierende systemische Entzündung - ein typisches Merkmal für entzündlich rheumatische Erkrankungen - ist mit einem hohen kardiovaskulären Risiko verknüpft und stellt zusätzlich eine Prädisposition für metabolische Krankheitsbilder und Sarkopenie dar.

Das metabolische Syndrom findet sich z. B. bei über $40 \%$ der Menschen mit einer rheumatoiden Arthritis (RA) im Vergleich zu ca. $25 \%$ in der Normalbevölkerung und ist in diesen Fällen Grund für einen mittleren bis schweren Krankheitsverlauf (DAS $>3,2$ ).

Zytokine aus den Fettzellen wie z.B. TNF-alpha, Interleukin-1 und Interleukin-6 führen zur erhöhten Insulinresistenz mit dem hohen Risiko der Entwicklung eines Typ-2-Diabetes. Mit dem gemeinsamen Vorliegen z.B. einer rheumatoiden Arthritis und eines Typ-2-Diabetes wird das kardiovaskuläre Risiko der Betroffenen deutlich verstärkt. Neben dem skizzierten Komorbiditätsrisiko bei Menschen mit entzündlich rheumatischen Erkrankungen verstärkt die häufig krankheitsbedingt ausgelöste Immobilität zusätzlich den Muskelabbau (Sarkopenie), die Entstehung eines Diabetes und die Entwicklung einer Arteriosklerose. So haben RA-Patienten ein um $60 \%$ erhöhtes Risiko für eine koronare Herzerkrankung und die kardiovaskuläre Mortalität ist im Vergleich zur Normalbevölkerung zusätzlich um $45 \%$ erhöht.

In diesem Zusammenhang ist das Aufzeigen des hohen Stellenwertes von sportlichen Aktivitäten als Therapiebaustein für verschiedene entzündich rheumatische Erkrankungen äußerst wichtig, da über die Aktivierung verschiedener Myokine (z.B. IL-6 und IL-15) in der Muskulatur nicht nur eine antiinflammatorische Wirkung beschrieben wird, sondern auch das Komorbiditätsrisiko erfolgreich reduziert werden kann. Diese aufgezeigten Zusammenhänge zwischen systemischer Entzündung, Komorbidität und des therapeutischen Nutzens von körperlicher Aktivität sind für uns Anlass genug, ein Schwer- punktheft zum Thema „Rheuma und Lifestyle" zu erstellen.

In ihrem Beitrag „Rheumatoide Arthritis und Sport" unterstreichen die Autoren Ostendorf, Schütt und Sewerin die Wichtigkeit einer regelmäßigen körperlichen Aktivität für Menschen mit einer rheumatoiden Arthritis. Es wird durch die körperliche Fitness nicht nur Wohlbefinden und Lebensqualität sowie die emotionale Befindlichkeit gefördert, sondern auch die Krankheitsaktivität und die fortschreitende Gelenkdestruktion positiv beeinflusst.

Die Autoren Andreica und Braun berichten in ihrem Beitrag „Spondyloarthritiden und Lebensstil" auch für diese Erkrankung über den wissenschaftlich belegten Nutzen regelmäßiger Übungen und körperlicher Aktivität. Das Interesse der Patienten an einer gesunden Lebensweise wie z.B. einer Nikotinentwöhnung und das Management der Gewichtskontrolle habe in den letzten Jahren zugenommen.

Die schädliche Wirkung des Nikotinkonsums konnten die Autoren auch für die axiale Spondyloarthritis darstellen.

Mit einer zweiten Veröffentlichung des Rheumazentrums Ruhrgebiet (Herne) in Kooperation mit der Rheumatologie der Technischen Universität Dresden beschreiben die Autoren Kiltz, Braun und Tausche den Zusammenhang von „Gicht und Lifestyle". Als wichtige Bausteine des therapeutischen Vorgehens wird eine rasche Behandlung der zugrundeliegenden metabolischen Ursachen mit Umsetzung einer bewussten Ernährung, der Einschränkung des Alkoholgenusses, einer dosierten Gewichtsabnahme sowie ausreichender körperlicher Aktivität beschrieben.

In seinem Beitrag „Ernährung bei rheumatischen Erkrankungen" stellt der Autor Keyßer den Zusammenhang zwischen Ernährung und dem Risiko, per se eine rheumatische Erkrankung zu entwickeln, her. So können einzelne Nahrungskomponenten wie z.B. Seefisch mit hohem Fettgehalt, Zitrusfrüchte oder geringe Al-

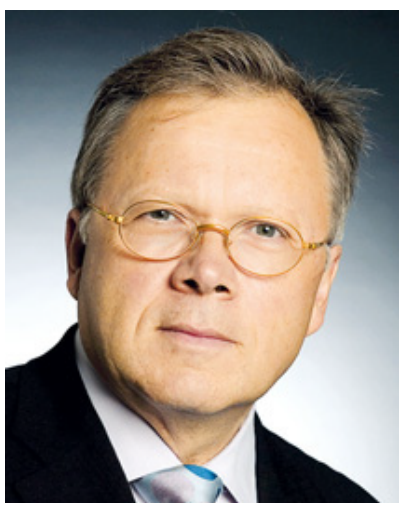

Prof. Dr. Heinz-Jürgen Lakomek

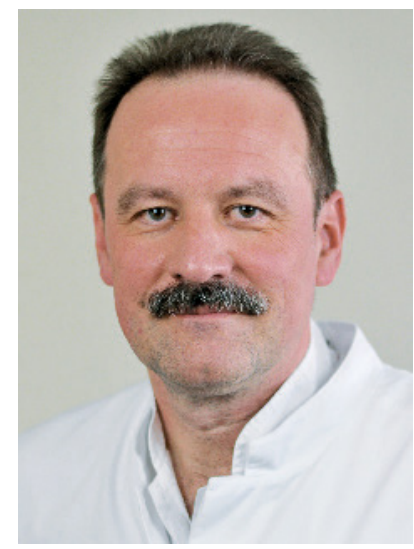

Dr. Roger Scholz 
koholmengen protektiv auf das RA-Risiko wirken.

Eine wichtige Klammer für die oben vorgestellten Beiträge stellt der Aufsatz von Pfingsten zum Thema „Chronische Schmerzerkrankung und Bewegung/Aktivität/Sport" aus Sicht eines Leitenden Psychologen einer universitären Schmerzklinik dar. Bei Betrachtung der Risiken durch verschiedene Aspekte in der Lebensform bei Rheuma wird durch diesen Beitrag der Einfluss von Schmerzen auf das Verhalten der betroffenen Menschen transparent dargestellt. So können Schmerzen zu Verhaltensmustern wie Inaktivität, Schonung und Vermeidung führen. Durch die Vermittlung verhaltenstherapeutischer Prinzipien in Patienteninformationen wie auch in der Ausgestaltung körperlicher Aktivierungsprogramme kann das Ablegen des Vermeidungsverhaltens gelingen.

Das Thema "Lifestyle-Änderung" hat für entzündlich rheumatische Erkrankun- gen wie auch für die Gicht einen hohen Stellenwert, da die systemische Entzündung nicht nur Komorbiditäten wie das metabolische Syndrom und den Typ2-Diabetes fördert, sondern zusätzlich in vielen Fällen Immobilität auslöst, was zur Potenzierung der Krankheitsfolgen aus den beschriebenen Komorbiditäten führt. Wie die Arbeitsgruppe von Prof. Ostendorf richtigerweise ausführt, bewegen sich $\mathrm{Pa}$ tienten z.B. mit rheumatoider Arthritis zu wenig, mutmaßlich auch aus der Annahme heraus, sportliche Aktivitäten könnten die Gelenkfunktion verschlechtern. Hier fordern die Autoren die grundlegende Multiplikation und Streuung von positiv aufklärenden Informationen und Empfehlungen zum Thema „Rheumatoide Arthritis und Sport für Arzt/Rheumatologe und Patienten in Klinik und Praxis“.

Mit dem vorliegenden Schwerpunktheft soll das Ziel der Multiplikation des Wissens um die positiven Effekte von Sport und ei- ner Änderung des Lebensstils bei Rheuma nachhaltig verfolgt werden, damit in Deutschland noch flächendeckender die Motivation zu mehr Bewegung, zur Adhärenz, zur Steigerung des Trainingsverhaltens sowie zur Kontrolle der erreichbaren Ziele und Funktionsverbesserungen breit gefördert wird.

Die Herausgeber dieser Ausgabe sind sich sicher, dass die vorliegenden Beiträge wichtige Einblicke und hilfreiche Erkenntnisse für die Versorgung von Menschen mit Rheuma vermitteln.

\section{Prof. Dr. med. Heinz-Jürgen Lakomek} Minden

Herausgeber

\section{Dr. med. Roger Scholz}

Oschatz

Gastherausgeber 